Résumés des conférences et travaux

\title{
Sources et histoire de la tradition sanskrite
}

Jan E. M. Houben

\section{(2) OpenEdition} Journals

Édition électronique

URL : https://journals.openedition.org/ashp/1988

DOI : 10.4000/ashp.1988

ISSN : 1969-6310

Éditeur

Publications de l'École Pratique des Hautes Études

\section{Édition imprimée}

Date de publication : 1 septembre 2017

Pagination : 289-297

ISSN : 0766-0677

\section{Référence électronique}

Jan E. M. Houben, «Sources et histoire de la tradition sanskrite », Annuaire de l'École pratique des hautes études (EPHE), Section des sciences historiques et philologiques [En ligne], 148 | 2017, mis en ligne le 03 octobre 2017, consulté le 06 juillet 2021. URL : http://journals.openedition.org/ashp/1988 ; DOI : https://doi.org/10.4000/ashp.1988 


\title{
SOURCES ET HISTOIRE DE LA TRADITION SANSKRITE
}

\author{
Directeur d'études : M. Jan E. M. HoubeN
}

Programme de l'année 2015-2016 : I. Introduction au sanskrit, lingua franca philosophique et scientifique (cours de master). - II. La maison védique selon les sources textuelles et rituelles. - III. Pāninini sans détour : thèmes grammaticaux, problèmes linguistiques, domaine de realia.

I. Non seulement la grammaire (vyākarana) et la médecine (āyurveda), mais aussi, comme nous l'avons vu l'année dernière, la métrique (chandas) étaient pertinentes pour tous les systèmes de philosophie et pour toutes les écoles de pensée qui, entre eux, s'attaquaient et se critiquaient sans cesse. Une bonne connaissance de la métrique (chandas) était indispensable pour chaque auteur et pour chaque lecteur et connaisseur de la poésie, séculière ou érudite, d'une inspiration brahmanique, bouddhiste ou jaina. Le terme sarvapārșadam ou sarvapārișadam «pertinent pour toutes les assemblées »a été utilisé dans les anciens commentaires pour la grammaire et pour la médecine indiennes. Le début du traité Chandonuśāsanam du polymathe jaina Hemacandra présente également la discipline de la métrique comme généralement pertinente pour les auteurs de la poésie, sans utiliser explicitement le terme sarvapārșadam ou sarvapārișadam. Pas très étonnamment, la métrique des textes védiques était exclut par Hemacandra. Par contre, le commentaire mrtasamjjìvin̄̄ de Halāyudha (date estimée : $\mathrm{x}^{\mathrm{e}}$ siècle), comme nous l'avons vu, justifia la nécessité d'étudier la métrique justement par sa pertinence pour le rituel védique, même si la métrique séculière est beaucoup plus proéminente dans son texte commenté, le Chandas-Śāstra de Pingala. Du point de vue de la méthode et des termes techniques, la métrique de Hemacandra est pourtant une continuation étroite de la discipline présentée dans l'ancienne œuvre de Pingala.

Nous étudiions le sanskrit versifié à travers un chapitre du Bhāgavata-Purāṇa sur le nārāyaṇa-varma " armure de Nārāyaṇa ». Le choix spécifique de ce passage est inspiré par les actualités de mi-novembre 2015, et plus généralement par le problème de la violence et la non-violence qui a intensément occupé les anciens indiens. Le chapitre 6.8 du Bhāgavata-Purāna traite d'un savoir (vidyā) protecteur qu'on croit pouvoir servir d' « armure » contre les attaques et les agressions des ennemis. Conceptuellement nous trouvons ici une continuation de l'acception réceptive d'ahimisa en tant qu' « absence de blessure (qu'on veut obtenir pour soi-même) », l'acception courante dans les plus anciens textes védiques (voir l'article de H. W. Bodewitz dans le volume Violence denied, sous la dir. de J. Houben et K. R. van Kooij, Leyde, 1999). À partir de la Chāndogya-Upaniṣad nous commençons de trouver cette autre acception de ahimsā qui est beaucoup mieux connue, la « non-violence envers les autres » comme règle de vie - une acception qu'on a à tort voulu lier à une dérivation du mot ahimsā d'un thème désidératif de han « tuer» (voir Mayrhofer 1976, 595 : dérivation de ahimsā de hinasti avec renvois bibliographiques pour la dérivation intenable de 
han). Métriquement, Bhāgavata-Purāṇa 6.8 est pour la plupart en ślokas simples, avec une partie centrale en Triștubh (Upajāti) et quelques lignes hypermétriques. D'autres exceptions métriques dérivent de la nécessité de donner une formule rituelle.

La ahims $\bar{a}$ comme règle de vie est mentionnée dans Yoga-Sūtra 2.35 ahimsāpratișthāyām tatsaminidhau vairatyāgah « Quand (le pratiquant du Yoga est) bien établi dans la non-violence, l'hostilité disparaît autour de lui ». La pratique de la non-violence - comme l'explique le plus ancien commentaire, la pratique d'entièrement éviter les pensées indésirables, les pensées violentes - est ici liée à un résultat extérieur, visible et tangible. Ce qui est remarquable est que le résultat extérieur mentionné n'est pas que le monde soit non-violent vers le pratiquant du Yoga, mais plutôt que l'hostilité disparaît entre les êtres vivants autour du pratiquant. Les commentaires confirment qu'il s'agit de l'hostilité entre « les êtres vivants » (sarvaprāṇinām, Yoga-bhāṣya), par exemple l'hostilité entre chats et souris (mārjāramūṣakādīnām, Vijñānabhikṣu). Que ce soit une croyance sans base empirique ou une observation réelle, on trouve en fait des idées parallèles ou plus ou moins comparables dans d'autres traditions, notamment dans le bouddhisme et même dans le Bible (voir mon article « To kill or not to kill the sacrificial animal...» dans le volume susmentionné, p. 141). La mention d'un lien entre pratique mentale et un résultat supposé à l'extérieur pourrait sembler un peu particulier, mais elle s'insère en fait assez bien dans la tradition du Yoga et surtout dans celle des systèmes jumelés de Yoga-Sāmkhya (à noter, par exemple, que le plus ancien commentaire sur le Yoga-Sūtra, le Yoga-Bhāṣya, se présente dans les colophons comme faisant partie d'un sāmkhyapravacana). Selon l'enseignement du Sāmkhya, qu'on attribue à Kapila, sage légendaire d'un passé lointain, le processus de création cosmogonique commence à un niveau subtil et « mental» et se développe jusqu'au niveau du monde extérieur. Un autre texte, le Baudhāyana-Gṛhya-Śeșa-Sūtra 4.16.4, mentionne une procédure pour se vouer à une vie ascétique qui contient la formule abhayam sarvabhūtebhyo mattas svāhā « de ma part : sécurité à tous les êtres, $s v \bar{a} h \bar{a} »$. La procédure est attribuée à un Kapila.

Tandis que des courants ascétiques représentés dans le Yoga-Sāmkhya et dans le bouddhisme et jainisme ont promu la non-violence comme règle de vie, les conflits armés étaient plutôt appréciés par d'autres. Citons dans ce contexte le mot rána du sanskrit védique, de la racine ran « réjouir », " se réjouir », dont le champ sémantique, couvrant à la fois « joie » et « bataille », peut être comparé avec la gamme d'ac-

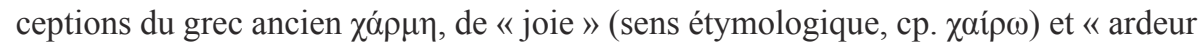
guerrière » à « lutte » (Beekes 2010 Etym. Dict. of Greek, vol. 2 p. 1606). Dans un passé moins éloigné, les concepts de la « guerre juste » (iustum bellum) et du droit de faire la guerre (ius ad bellum) ont été mis au point aux époques où les guerres étaient toujours les affaires des guerriers et les armes de guerre ne pourraient tuer que quelques personnes, ou même un nombre considérable de personnes. Ces concepts perdent leur validité quand des armes de guerre sont disponibles qui peuvent détruire, même plusieurs fois, tous les êtres humains ou toute la vie sur la terre. C'est pourquoi les justifications ou même les glorifications de la violence guerrière de l'Antiquité ne peuvent pas être étroitement transposées vers la modernité. Sous la force des circonstances, le droit de faire la guerre est alors remplacé par une obligatio pacis, une obligation de maintenir la paix. Cette obligation devrait appartenir tout d'abord aux 
grands pouvoirs politiques qui disposaient ou s'arrogeaient, auparavant, du ius ad bellum, mais aussi aux grandes traditions religieuses, parmi lesquelles les religions missionnaires qui acceptent leurs adhérents non seulement par hérédité ou par tradition, mais aussi par conversion. Le Bouddhisme était selon les sources disponibles la première religion missionnaire qui entra dans le monde méditerranéen, avec l'envoi des ambassadeurs religieux par le roi Aśoka de l'Inde, autour de 250 avant notre ère. Selon ses propres édits sur rocher, Aśoka les envoya dans toutes les directions à partir de son royaume, aussi vers l'extrême Ouest : aux rois Antiochos (II Theos de Syrie), Ptolémée (II Philadelphe d'Égypte), Magas (de Cyrène, actuellement en Libye), Antigone (Gonatas de Macédonie) [J. Bloch, Les inscriptions d'Aśoka, Paris, Les Belles Lettres, 1950, p. 129-131]. La mission religieuse bouddhiste, par la prédication et par l'exemple, était non-violente, ce qui n'a pas toujours exclu la participation à la guerre des laïcs et des pays bouddhistes (L. Schmithausen, «Aspects of the Buddhist attitude towards war » dans le volume susmentionné, Violence denied). La mission religieuse, tout à coup introduit dans la Méditerranée par le bouddhisme, réapparaît dans le christianisme ancien, de façon entièrement non-violente dans ses premières siècles; ensuite dans le plus ancien Islam, où il était lié au concept du jihâd, l'« effort » religieux, dont on distingue un jihâd majeur, la guerre de l'âme spirituelle contre les ennemis intérieurs, et un jihâd mineur, la guerre contre les ennemis extérieurs, ce qui peut inclure la lutte armée défensive ou offensive. De nouveau, les concepts de base étaient acceptés et interprétés dans les premiers siècles de l'islam quand les armes de destruction modernes n'étaient ni disponibles ni imaginables. Pour conclure cette exploration du principe proposé dans le Yoga-Sāmkhya et dans d'autres traditions indiennes et non-indiennes - victoire intérieure sur les pensées indésirables et paix à l'extérieur prévu comme résultat - nous le résumons en composant le śloka suivant :

yasyāhimiāpratișthāsti tyajyate tasya saminidhau/ vairas tathä ca loke'smin heyaì duḥkham anägatam //

II. Dans notre étude des rites dans et autour de la maison védique, nous nous concentrions cette année sur le Sāmavidhāna-brāhmaṇa, un texte qui appartient, au moins en partie, au genre des Brāhmanasas qui, au sens large, inclut celui des Āraṇyakas et celui des Upaniṣads. Le Sāmavidhāna-brāhmaṇa appartient aux écoles du Sāmaveda, le Veda du chant. Dans la deuxième moitié du XIX ${ }^{\mathrm{e}}$ siècle ce texte attira beaucoup l'attention, dans le contexte des théories qui venaient d'être formulées sur la pensée magique et sa place dans le développement de l'humanité, car il semblait convenir parfaitement à une approche évolutionniste dans l'histoire de l'humanité, qui était en vogue à cette époque. Après un oubli de presqu'un siècle, ce texte a fait l'objet d'une thèse de doctorat sous ma direction par $\mathrm{M}^{\mathrm{me}}$ A.-M. Quillet, soutenue mi-décembre 2015.

Le Sāmaveda, le «Savoir de Chansons », s'est spécialisé dans les mélodies sacrées qui doivent être chantées lors des rituels védiques. Le Sāmaveda est transmis jusqu'à nos jours, dans des écoles spécialisées en Inde, comme le plus ancien et, à plusieurs égards, le plus important des textes védiques, le Ragveda. Tant pour les étudiants que pour les chercheurs, j'ai mis en ligne quelques minutes d'un enseignement 
du R.gveda, le savoir des hymnes, en Inde : https://vimeo.com/82963699; et quelques minutes d'un enseignement du Sāmaveda : https://vimeo.com/90023730; tous les deux enregistrés lors de mon travail de terrain en Inde.

Huit textes d'exégèse canonique (Brāhmaṇas) sont traditionnellement attribués au Sāmaveda et font partie de sa littérature révélée. Le Sāmavidhāna-brāhmaṇa est le troisième dans la liste traditionnelle. Nous avons étudié le genre du « Brāhmaṇa » dans nos cours de 2004-2005 jusqu'à 2008-2009, quand les Brāhmaṇas du Sāmaveda, plus spécifiquement le Sāmavidhāna-brāhmaṇa, était notre sujet. En fait, seulement les deux premières textes que la tradition du Sāmaveda appelle Brāhmaṇa, le Tāṇḍya-mahābrāhmaṇa ou Pañcavimía-brāhmaṇa et le Șaḍvimśa-brāhmaṇa, représentent pleinement le genre du Brāhmaṇa : un texte en prose védique qui procède par des discours et des narrations et qui donne des analyses selon plusieurs points de vue du grand rituel « solennel » ('srauta) dans lequel sont utilisés les hymnes et les formules de la grande collection, la Samhitā de l'école concernée : Ṛgveda, Sāmaveda, Yajurveda ou Atharvaveda. D'ailleurs, nous avons vu (résumé des conférences de 2013-2014) que le Șaḍvimśa-brāhmaṇa convient en fait assez bien au genre plus spécifique des Āraṇyakas. Les premières importantes études du genre du Brāhmaṇa sont La doctrine du Sacrifice dans le Brāhmaṇa, Paris, 1898, par Sylvain Lévi; Die Weltanschaung der Brāhmana-texte: Vorwissenschaftliche Wissenschaft, Göttingen, 1919, par Hermann Oldenberg. Des citations du Sāmavidhāna-brāhmaṇa sont à plusieurs reprises utilisées et discutées dans ces deux études. Pourtant, il a le caractère d'un Brāhmana seulement dans le mythe d'introduction, dans le premier chapitre du premier livre. Dans le reste, le caractère du texte est plutôt comparable avec celui du Ṛgvidhāna, qui prescrit l'usage des stanzas et des hymnes du Ṛgveda dans des applications rituelles selon plusieurs contextes et objectifs. Selon l'éditeur anglais du Sāmavidhāna-brāhmaṇa, A. C. Burnell (1873, xII et suiv.) : « Ce livre a sa valeur indépendante, dans la mesure où il préserve pour nous une image du début d'une civilisation, et des idées et des pratiques que d'autres nations ont rejetées ou cachées de honte dans le cadre de leur progrès, et qui n'existent actuellement à peu près nulle part sur la terre. » Auguste Barth dans son compte rendu de l'édition de Burnell commente l'attribution du Sāmavidhāna-brāhmaṇa à « l'âge fétichiste » : « ... les divisions d'Auguste Comte, si commodes qu'elles soient pour marquer à grands traits les cadres de l'histoire, ne sont pas le moyen le plus sûr d'arriver à une appréciation juste et fine des faits particuliers du passé ».

Selon le Sāmavidhāna-brāhmaṇa les chants du Sāmaveda, qui doivent être chantés par une personne bien formée et bien préparée, ont plusieurs objectifs, par exemple, obtenir des biens ou une guérison, enlever les effets des mauvais rêves, voire des rêves prémonitoires. Ce texte peut-il être compris correctement et exclusivement comme un texte de magie? Le résumé du cours de 2008-2009 discute quelques aspects de ce problème et un article en développe plusieurs points (Houben 2009 : "A song against bad dreams: magic, superstition or psychology? », contribution au colloque Le Sommeil et les rêves dans le monde indien, actes paru dans The Indian Night, Delhi, sous la dir. de Claudine Bautze-Picron). Il est à noter que la lecture «magique » d'un texte comme le Sāmavidhāna-brāhmaṇa, même si elle reste attrayante pour un grand nombre de passages, présuppose, en fait, un réalisme naïf comme point de repère chez ceux qui 
acceptent le système rituel. Or, un tel réalisme naïf est justement ce qui est explicitement exclu dans les premiers paragraphes du Sāmavidhāna-brāhmaṇa, qui décrivent une création séquentielle du monde, à partir du Brahman (neutre), à travers le dieu suprême Brahmā (masc.) dont l'esprit devient Prajāpati, le « seigneur des créatures ».

Dans les sections qui suivent cette « ouverture » significative, le Sāmavidhānabrāhmaṇa présente des procédures ascétiques appelées krcchra « dures », qui visent à rendre le pratiquant pur et capable de réaliser les procédures d'autres section et chapitres : les procédures pour obtenir des résultats équivalents à ceux des grands rites solennels (les yajña souvent difficiles à exécuter comme le sacrifice de Soma); les prāyaścitta " expiations " pour toutes sortes d'erreurs; les kāmya, à l'occasion d'un souhait ou d'un vœu particulier. Les procédures visent toute une liste de choses désirables : un miroir de la société contemporaine des adhérents du Sāmaveda et du Sāmavidhāna-brāhmaṇa. Le terme krcchra renvoie ici à une forme de tapas « austérité » (vol. I, p. 228), et paraît dans l'Āyurveda dans le composé mūtra$k r c c h r a$, qui renvoie à une émission difficile ou douloureuse d'urine, dysuria. Les Dharmaśāstra connaissent des procédures ascétiques sous plusieurs noms (le Manusmṛti l'appelle Prājāpatya), qui sont presqu'identiques aux krcchras du Sāmavidhānabrāhmaṇa, sauf qu'elles n'ont aucun caractère sāmavédique et ne sont pas discutées de façon très systématique.

Parmi les caractéristiques remarquables du Sāmavidhāna-brāhmaṇa se trouve le style « sūtra » dans lequel la brièveté est réalisée par des techniques particulières. Ici, ce style tellement important pour le sanskrit scientifique et philosophique est encore peu développé. Les phrases sont, d'abord, souvent elliptiques de façon systématique : un ou plusieurs mots sont à compléter, plus précisément, à continuer de la phrase qui précède immédiatement; parfois un mot doit être continué dans plusieurs phrases consécutives. Une forme développée de cette procédure se trouve dans la grammaire de Pāninin et est appelée anuvṛtti. Deuxièmement, il y a des renvois à un passage spécifique par une expression précise, par exemple etena kalpena « par la même procédure rituelle ». Les ellipses se laissent déterminer sur la base de la logique du texte; elles sont normalement confirmées et rendues explicites dans les commentaires de Sāyaṇa et de Bharatasvāmin. Troisièmement, le renvoi aux Sāman « mélodies » spécifiques qui doivent être utilisées dans les chants prescrits pour des procédures rituelles spécifiques se fait à travers le pratīka « visage » (dérivation thématisée du degré faible de praty-añc, Renou, 1952, Grammaire de la langue védique, p. 180). Celui-ci consiste en les premiers mots de la strophe sous-jacente de la mélodie, qui sont parfois joints dans un composé dont la fin est thématisée ou pourvue d'un suffixe tel que -īya-. La procédure pour transformer les premiers mots d'un passage en composé avec une fin thématisée remonte au sanskrit védique, un exemple bien connu étant yeyajāmahăh dans le Vājasaneyi-samhitā, qui renvoie aux "formules en yé yájāmahe » (Renou 1952, Grammaire de la langue védique, p. 141). Certains composés tels que iti-ha-āsa- sont continués comme des mots fréquents dans le sanskrit classique. Une excellente discussion sur le phénomène se trouve déjà dans Altindische Grammatik de Jakob Wackernagel, vol. II,1, Einleitung zur Wortlehre: Nominalkomposition, p. 325, où les composés sont discutés, qui « aus beliebigen Sätzen oder Satzstücken, oft mit willkürlicher Zustutzung der Wörter, zusammengeschweisst sind » (les exemples 
mentionnés, à part ye-yajāmahá du VS, incluent āpo-revatyai « der Strophe ápo revatīh... "; hotā-yakșa « der Praișa hotā yakșat », cameșu pațyate " wird in den Sprüchen mit ca me ... aufgeführt » (Mahābhāṣya sous AA 5.2.4 Vārtt. 2). A camakaet camaka-sūkta (i.e., TS 4.7.1-11, passage consistant en formules contenant les mots ca ma) mentionnés par Wackernagel, Altindische Grammatik, vol. II,2, p. 144, qui sont aussi trouvables dans le grand dictionnaire dit « de Saint Petersbourg » de Böhtlingk et Roth, on peut ajouter namaka (i.e., TS 4.5 : les strophes contiennent le mot namas, hymne aussi connu sous le nom de Rudrādhyāya, Rudropanișat, Śatarudrīya), qui n'est expliqué dans aucun de ces dictionnaires. Wackernagel, Altindische Grammatik, vol. II,1, p. 326, renvoie également à d'autres discussions pertinentes sur ce sujet, y compris justement l'introduction de A. C. Burnell à son édition du Sāmavidhāna-brāhmaṇa ou l'emploi du pratīka est fréquent et bien développé. La procédure donne des renvois brefs et suffisamment exacts pour ceux qui sont parfaitement familiers avec les textes principaux d'une tradition. Dans la pratique, la procédure présuppose que l'étudiant connaisse déjà par cœur la collection d'hymnes et de chants, la Samihitā, de son école. La procédure permettant de joindre les premiers mots d'un passage pour y renvoyer de façon efficace est aussi attestée dans l'Avesta, comme l'a déjà fait remarquer Wackernagel. Par exemple, vahištōišti- renvoie au Yasna 53 qui commence par vahišstā ištiš..., spantā-mainyū- au Yasna 47 qui commence par spəntāmainy $\bar{u} . .$. La procédure remonte donc sans doute à l'époque indo-iranienne, mais elle est tellement « naturelle » qu'elle n'est pas nécessairement spécifique aux traditions indo-iraniennes.

Dans le Sāmavidhāna-brāhmaṇa nous étudiions en particulier SVB 3.3.6-7 avec le commentaire de Sāyaṇa (attribué à Sāyaṇa). Le passage commence par athāto vāstupraśamanam « ensuite, donc, l'apaisement de la demeure ». Sten Konow, 1893, traduit « Jetzt folgt, wie er das Haus weiht». Mais pour renvoyer à une « Weihung » le sanskrit a d'autres termes, par exemple abhișeka, et de toute façon ni « Weihung » ni abhiṣeka ne couvrent la procédure développée dans le reste du passage. Sāyaṇa explique vāstupraśamanam correctement : vāstu nūtanagṛāadhișthānam, tasya śamanam tatratyarakṣahprabhṛtīnām nivāranam ity arthah. " vāstu (c'est à dire) l'endroit de la maison actuelle; l'apaisement de cela (c'est-à dire) la protection contre les démons (rakṣas), etc. qui y résident ». Est-ce que vāstu renvoie à la maison déjà construite ou au chantier où on va construire la nouvelle maison? Le mot devrait couvrir les deux acceptions, car le texte prescrit que la procédure est répétée chaque quatre mois ou chaque année : même si le site est encore vide la première fois, il serait déjà pourvu d'une maison la deuxième fois - au début une construction extrêmement simple voir primitive, sans briques ni pierres. Comme le faisait remarquer Louis Renou, « La maison védique », Journal asiatique 231, 1939, 504 : avec la maison védique « nous sommes donc en présence d'un type de maison extrêmement rudimentaire, composé d'une armature de poteaux reliés au sommet par des poutres transversales sur lesquelles est fixée une couverture de chaume. Les cloisons sont faites de nattes »; à ceci il ajoute une autre remarque toujours valide : " Le phénomène apparaît dans toute sa singularité aujourd'hui où nous pouvons mesurer à quel haut degré de technique architecturale étaient parvenues certaines civilisations préhistoriques dans l'Inde du Nord-Ouest $»$. 
III. Parmi les faits de la réalité du grammairien Pāṇini il y a son environnement naturel, économique et politique. Au deuxième millénaire avant notre ère, le Nord du sous-continent indien était une terre féconde et encore largement boisée, traversée par sept grandes fleuves. Nous y voyons l'émergence d'un complexe culturel, rituel et textuel, que l'on appelle « védisme ». Un millénaire plus tard, au milieu du première millénaire avant notre ère, le védisme s'est répandu dans tout le Nord du sous-continent indien, de la région des sept fleuves dans le Nord-Ouest vers l'est du bassin du Gange, et au delà jusqu'au Bengale.

Tout un complexe de rituels préexistant est présupposé dans les hymnes du Rgveda. Les objets et les circonstances auxquels ils renvoient montrent qu'il devrait dater d'avant l'âge du fer, qui, sur le sous-continent indien, commence vers le $\mathrm{x}^{\mathrm{e}}$ siècle avant notre ère. Il y avait d'autres complexes de rituels et de textes, mais celui du védisme est le seul qui a survécu jusqu'à nos jours. Comment le védisme a-t-il pu parvenir à ceci? Le complexe culturel, rituel et textuel du peuple védique contenait des éléments qui convenaient à un contexte d'agro-pastoralisme, ce qui, à son tour, convenait à l'environnement de l'époque. Non seulement le complexe rituel avait-il trouvé une bonne « niche » dans l'Inde du Nord de cette époque dans laquelle il pouvait se répandre, le peuple védique développait aussi des techniques spéciales pour enseigner et transmettre ce complexe rituel avec la plus grande précision. Le succès de l'agro-pastoralisme avec lequel le rituel védique était associé transformait, au cours des siècles, ce même environnement en un espace largement cultivé et urbanisé. Grâce aux techniques extraordinaires pour transmettre les bases textuelles et rituelles du védisme, ce complexe a ensuite réussi à survivre même au delà de son époque et de sa niche écologique originales.

Comment les Vaidika, ceux qui étudient le Veda et exécutent les rituels védiques, ont-ils transmis ce texte oralement à travers les millénaires avec une remarquable précision, mot par mot, syllabe par syllabe et même phonème par phonème? Une discipline auxiliaire, la grammaire, contribuait à la précision de transmission, et garantissait non seulement la maîtrise et la bonne compréhension des textes védiques, mais définissait aussi un haut standard de la langue parlée encore très proche du langage du Veda, une langue en usage à l'époque de Pāṇini, ca 350 avant notre ère, qui s'est imposée au cours des siècles suivants comme une lingua franca pour des diverses disciplines telles que les mathématiques et la médecine. Ensuite, sur les traces de Panini et de ses prédécesseurs, des grammaires simplifiées sont parues. Une langue parlée devenait ainsi, pour la première fois dans l'histoire de l'humanité, accessible pour un plus grand public par une description très nuancée et fiable. La perfection et l'économie de la science de la grammaire étaient telles qu'elle s'imposa comme discipline paradigmatique pour toutes les autres sciences. Les mathématiques en Inde, d'un très haut niveau, restaient en service des savoirs rituels, religieux et humanistes qui les encadraient et qui étaient renforcés et stabilisés par leurs contributions. Ironiquement, la grammaire devenait non seulement un outil de stabilisation pour le complexe rituel et textuel du Veda et les communautés associées, mais aussi un instrument d'expansion et de stabilisation pour un nouveau complexe rituel-textuel qui commençait dans la région graduellement cultivée et urbanisée du bassin du Gange dans les siècles avant le début de notre ère, le bouddhisme. Les auteurs des textes hors $\mathrm{du}$ 
cercle initial commençaient à préférer le sanskrit et contribuaient ainsi à la richesse $\mathrm{du}$ « monde » sanskrit, qui, lui, était stabilisé par une description grammaticale remarquablement efficace. La grammaire garantissait la compréhension entre les instruits dans des coins les plus éloignés du sous-continent indien, à travers les siècles, et à partir du début de notre ère, aussi dans des très grandes parties de l'Asie centrale, de l'Asie du Sud-Est et de l'Indochine.

À une plus large échelle, l'histoire nous permet de comparer trois variables pour plusieurs grandes civilisations : (a) «monde " culturel, qui semble universel mais qui est en fait soumis à des conditions limitatives, les « limites de la langue » (Wittgenstein) principale dans l'aire culturelle-linguistique concernée; (b) les outils pour apprendre et enseigner cette langue, tel que la grammaire; et (c) la « civilisation» colorée et projetée par la langue et la littérature. Voir dans ce contexte l'étude phare de Kees Versteegh, 1986, « Latinitas, Hellenismos, 'Arabiyya », Historiographia Linguistica 13.2-3:425-448.

Le parallélisme s'exprime dans ce tableau :

« monde » latin - grammaire latine - latinitas,

« monde » hellénique - grammaire grecque - hellenismos,

« monde » sanskrit - grammaire sanskrite - sanskrti,

« monde » arabe - grammaire arabe - arabiyya,

peut être invoqué pour souligner l'apport central de la grammaire pour le « monde » culturel, religieux et scientifique correspondant. La quatrième ligne renvoie ici à cette grammaire remarquablement parfaite de l'arabe de la fin du VIII ${ }^{\mathrm{e}}$ siècle, la grammaire de Sibawayhi à laquelle en renvoie par al-Kitaab, en relation avec le « monde » arabe et avec l' " arabiyya », la « civilisation » arabe qui doit inclure une maîtrise parfaite de la grammaire. Géographiquement et dans le domaine de la phonétique on peut soupçonner un lien avec la riche et ancienne tradition grammaticale de l'Inde, connue en Perse depuis des siècles.

Serait-il alors possible de décrire le succès indéniable du « monde » sanskrit dans le premier millénaire comme un succès dans l'idéosphère de l'Inde et des régions voisines? Qu'est-ce qu'il s'est passé aux chevauchements de deux ou plusieurs idéosphères?

Aidé par son association avec des systèmes rituels, au début le système des rituels védiques, le sanskrit s'est répandu dans le sous-continent indien. La grammaire a contribué à la propagation du sanskrit et à sa stabilisation linguistique. Le sanskrit paraît donc en tant qu'une langue classique non seulement avec une pertinence nationale, indienne, mais d'une importance mondiale et universelle, tout comme d'autres langues classiques du monde (bhülokabhāṣa) telles que le grec ancien, le latin et le chinois classique. En conséquence, j'ai composé la strophe suivante pour souligner la tendance vers l'universalité du sanskrit, tendance qu'on trouve déjà dans le sanskrit de l'époque védique, comme le proposaient Louis Renou et Wilhelm Caland. Le sanskrit védique connaissait des « sociolectes » et des variations dans la transmission textuelle des écoles plutôt que des « dialectes » selon des lignes géographiques.

Commençant par une référence à la déclaration védique nālpe sukham asti, bhümaiva sukham (Chāndogya Up. 7.23.1), la strophe souligne la beauté phonétique du 
sanskrit, son importance dans la logique et d'autres systèmes de connaissances, et sa capacité à fournir des visions littéraires $(d h \bar{\imath})$ et les émotions brillantes (rājadrasā) à tous les lecteurs, bref, sa capacité à inspirer, élever et cultiver (samiskāriniñ) ses étudiants. La strophe est composée dans le mètre Mandākinī, mètre simple et fort mais pas très commun, qui suit la formule métrique ta-ma-ya-ra-ta-ga avec quatre caesurae (Mandāramarandacampū, p. 18 ligne 14 : mandākin̄̄ tmayartā go vedair vedayatir bhavet), équivalent à $4 \times($ ta-ga), soit quatre fois ta-ga.

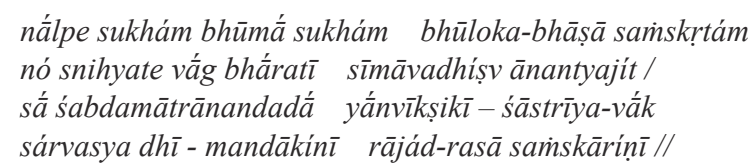

Nous avons étudié, en outre, durant l'année, les technicités de la grammaire, surtout les métarègles et « règles du pouce » dans l'art de la grammaire pāṇinéenne, et, à cause de l'intérêt spécifique des étudiants, nous avons aussi étudié le Dhātupātha, la liste des racines, de la grammaire. Le 8 et le 15 avril 2016, $\mathrm{M}^{\text {lle }}$ Béatrice Bonino, préparant un mémoire de master 2 sous ma direction (soutenu le 24 juin), donna deux présentations sur le Dhātupāțha et la linguistique. 